EDITOR'S

CHALLENGES

FOR OPEN SCIENCE

\title{
REMAT: PERSPECTIVAS QUANTO À MIGRAÇÃO PARA O OPEN JOURNAL SYSTEMS 3
}

\author{
REMAT: Perspectives on migration for Open Journal Systems 3
}

\author{
Glauciane Klein Burgiert Padilha \\ Instituto Federal de Educação, Ciência e Tecnologia \\ do Rio Grande do Sul (IFRS) \\ Greice da Silva Lorenzzetti Andreis \\ Instituto Federal de Educação, Ciência e Tecnologia \\ do Rio Grande do Sul (IFRS)
}

\author{
Katia Arcaro \\ Instituto Federal de Educação, Ciência e Tecnologia \\ do Rio Grande do Sul (IFRS) \\ Daiane Scopel Boff \\ Instituto Federal de Educação, Ciência e Tecnologia \\ do Rio Grande do Sul (IFRS)
}

\section{COMO CITAR}

PADILHA, Glauciane Klein Burgiert et al. REMAT: perspectivas quanto à migração para o Open Journal Systems 3. In: ABEC MEETING, 2019, Fortaleza. Anais... São Paulo: Associação Brasileira de Editores Científicos, 2019. http://dx.doi.org/10.21452/abecmeeting.2019.217

\section{RESUMO}

Este artigo tem por objetivo historicizar a trajetória da REMAT: Revista Eletrônica da Matemática (e-ISSN 2447-2689) com o registro das últimas ações de melhoria realizadas em 2019. Essas incluem a ampliação do número de indexadores com metadados da REMAT, a migração do sistema de gestão do fluxo editorial e publicação de periódicos científicos na web, passando do Open Journal Systems (OJS) 2 para o OJS 3, e o depósito de metadados dos artigos da revista no sistema Digital Object Identifier (DOI). Com sustentação analítica em Lancaster (1995), Costa (1996), Harnad (1996), Campanario (2002), Patrus et al. (2016) e Brito et al. (2018), descreve-se a forma na qual os processos de editoração são realizados e projetam-se as melhorias que podem ser obtidas por meio do upgrade do sistema OJS. A partir dessas ações espera-se um melhor rendimento do fluxo editorial, facilitando o processo de submissão e editoração de artigos para autores, avaliadores e editores. Essas iniciativas têm por objetivo fazer com que a REMAT continue crescendo em termos de visibilidade e, principalmente, de qualidade. 
PALAVRAS-CHAVE

REMAT. Migração de Sistema. OJS 3. Indexadores. DOI.

\section{ABSTRACT}

This article aims to historicize the trajectory of REMAT: Revista Eletrônica da Matemática (e-ISSN 2447-2689) with the record of the last improvement actions carried out in 2019. These include the expansion of the number of indexers with REMAT metadata, migration of the system of editorial flow management and publication of scientific journals on the web, moving from Open Journal Systems (OJS) 2 to OJS 3, and the metadata deposition of journal articles in the Digital Object Identifier (DOI) system. With analytical support in Lancaster (1995), Costa (1996), Harnad (1996), Campanario (2002), Patrus et al. (2016) and Brito et al. (2018), it describes the manner in which the publishing processes are carried out and designs the improvements that can be achieved by upgrading the OJS system. From these actions, a better performance of the editorial flow is expected, facilitating the process of submission and editing of articles for authors, reviewers and editors. These initiatives aim to make REMAT continue to grow in terms of visibility and, especially, quality.

\section{KEYWORDS}

REMAT. System Migration. OJS 3. Indexers. DOI.

\section{INTRODUÇÃO}

A REMAT teve início em 2015 e tem como objetivo publicizar produções originais com enfoque em três seções: Matemática em Contextos Técnicos e/ou Tecnológicos, Ensino de Matemática e Matemática Pura e/ou Aplicada, bem como divulgar pesquisas sobre o uso de tecnologias digitais e suas contribuições para esses campos do conhecimento. Como um meio de divulgação científica periódico exclusivamente eletrônico, a REMAT enquadra-se no que Lancaster (1995) define como jornal eletrônico e, como tal, pode oferecer alguns recursos a mais do que os meios impressos oferecem, como mecanismos de busca e até mesmo manipulação de dados. Dentre as vantagens dos jornais eletrônicos, Lancaster (1995) destaca publicações mais rápidas de resultados de pesquisa e fácil comunicação entre pesquisadores e leitores. Nesse sentido, a REMAT vem investindo na missão de compartilhar com qualidade e eficiência (VISSIRINI et al., 2019) produções científicas que envolvem práticas educativas e resultados de pesquisas relacionadas à Matemática. Garantindo acesso livre às suas publicações semestrais, a Equipe Editorial entende a importância de difundir os achados científicos e as experiências educativas de sucesso não só na esfera acadêmica, mas usufruir da vantagem do meio eletrônico para disponibilizar à comunidade em geral o que vem sendo pensado e desenvolvido na academia.

Além da facilidade de acesso e disseminação que o jornal eletrônico proporciona, Lancaster (1995) já entrevia, há quase 25 anos, que esse meio viria a oportunizar maneiras inovadoras de apresentar os resultados de pesquisa, como o uso de animações, sons, links entre fontes, entre outros. Embora não citadas diretamente pelo autor, dentre as possibilidades que o meio impresso comum não dispõe, estão as ferramentas de acessibilidade. Nesse contexto e com vistas a 
ampliar o alcance da divulgação científica, a REMAT também vem investindo na acessibilidade digital (SILVA et al., 2018).

Outra vantagem do jornal eletrônico, segundo Lancaster (1995), é a facilidade no processo de avaliação por pares e de comunicação entre autores, editores e avaliadores. Essa questão do processo de avaliação por pares e de seus problemas intrínsecos é bastante discutido já há algum tempo (HARNAD, 1996; COSTA, 1996; CAMPANARIO, 2002); no entanto, é a metodologia de avaliação de produções científicas que perdura, sendo a mais utilizada atualmente (PATRUS et al., 2016) e, mais do que isso, "já há algum consenso de que o julgamento pelos pares [...] pode ser implementado nos meios eletrônicos, emprestando ao periódico eletrônico o mesmo reconhecimento do periódico impresso" (COSTA, 1996, p. 5). Auxiliando a dinâmica desse processo que, mesmo facilitado, segue sendo complexo e intrincado na medida em que há uma real preocupação com a ética, a imparcialidade e a qualidade do material a ser publicado, surgem os sistemas de gerenciamento e publicação de revistas eletrônicas. Entre os de acesso livre destaca-se o Open Journal Systems (OJS) que, só no Brasil, conta com mais de mil e setecentas revistas usuárias da ferramenta (BRITO et al., 2018).

Desde a sua criação até então, a REMAT vinha fazendo uso do OJS 2, que intermediava integralmente todo o processo de submissão, comunicação entre autores e editores, e entre esses últimos com avaliadores, até a edição final e, então, a publicação. Recentemente a instituição fomentadora ${ }^{1}$ da REMAT optou por migrar para a versão 3 do OJS e realizar a atribuição do DOI para as publicações do Portal de Periódicos do IFRS, no qual a REMAT inclui-se. Esta comunicação tem por objetivo descrever as perspectivas de melhoria da revista e das novas possibilidades de avanço frente às inovações do sistema.

\section{INDEXADORES E VISIBILIDADE}

A REMAT é um periódico indexado em bases de dados referenciais, nas quais seu conteúdo temático é descrito. São fornecidas informações dos artigos, tais como: autor e título do artigo, título e local de publicação do periódico, volume, número, e as páginas onde se encontra o artigo. Essas bases permitem a recuperação e ampliação da visibilidade dos títulos indexados, assim como atribuem ao periódico um valor técnico, tendo em vista que as informações publicadas na REMAT passam por uma avaliação criteriosa, dando ao periódico maior credibilidade.

A seguir, apresentam-se indexadores e buscadores em que a REMAT está presente, por ordem de cadastro.

a) Google Acadêmico;

b) Diretório de Políticas Editoriais das Revistas Científicas Brasileiras - Diadorim;

c) Instituto Brasileiro de Informação em Ciência e Tecnologia - ibict oasisbr;

d) Revistas de livre acesso - LivRe;

e) Sumários de Revistas Brasileiras - sumários.org;

\footnotetext{
1 Instituto Federal de Educação, Ciência e Tecnologia do Rio Grande do Sul (IFRS).
} 
f) Google Analytics;

g) Directory of open access journals - DOAJ;

h) Portal de Periódicos CAPES;

i) Diretório do Sistema Latindex: Sistema Regional de Información en Línea para Revistas Científicas de América Latina, el Caribe, España y Portugal.

De acordo com o Relatório de Acessos da REMAT gerado pelo OJS, foram contabilizados 34.792 acessos aos resumos e 54.362 acessos aos arquivos PDF/A de 2015 a 31 de agosto de 2019. No Gráfico 1 pode-se observar a evolução neste número de acessos por períodos. Em 2019 a REMAT foi incluída no Portal de Periódicos da Capes e no Latindex, o que impulsionou o número de acessos no último semestre, como observa-se no Gráfico 1.

Gráfico 1. Número de acessos aos resumos e arquivos PDF/A dos artigos da REMAT.

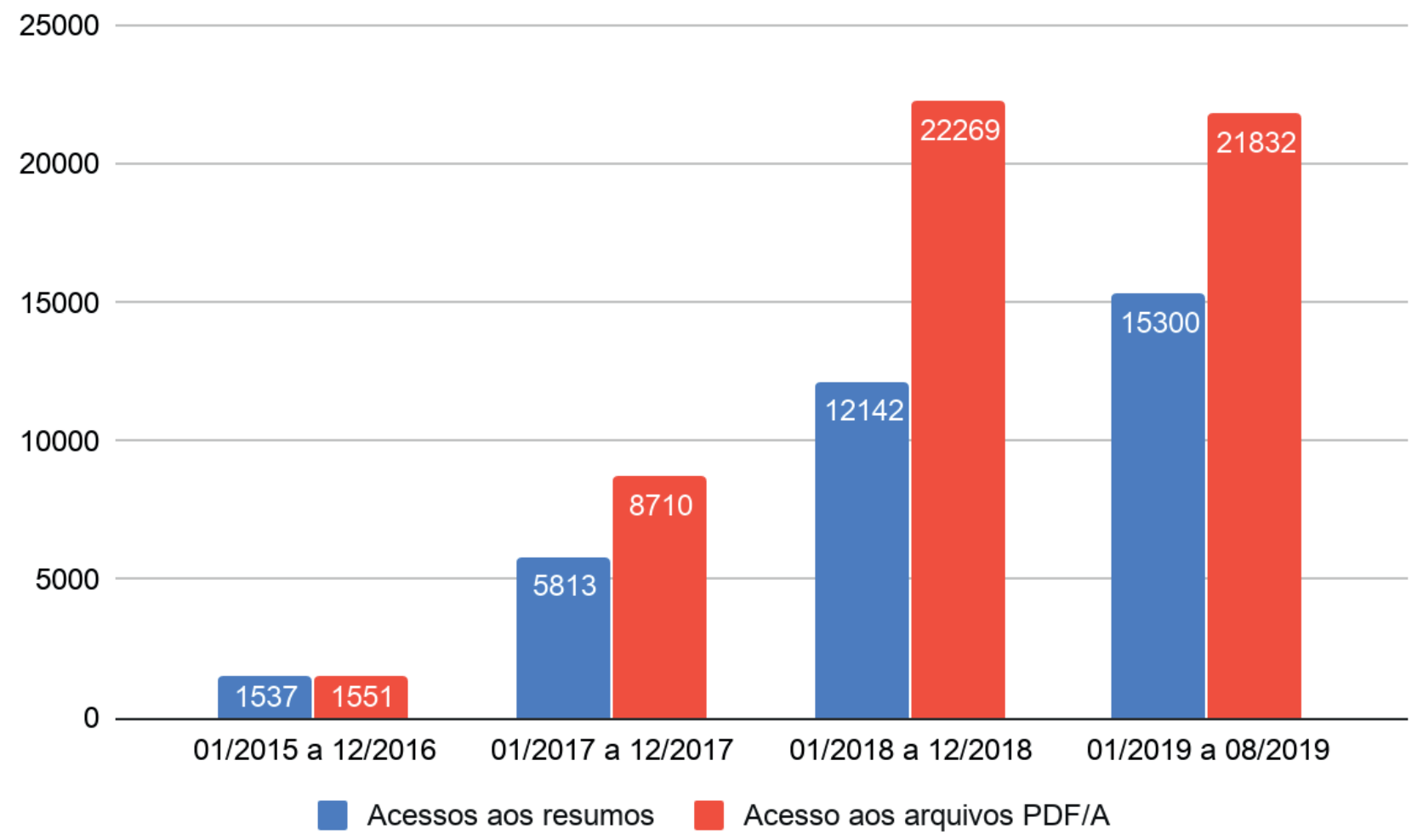

Fonte: Dados do Relatório de Acessos da REMAT de 2015 a 31 de agosto de 2019.

\section{MIGRAÇÃO PARA O OJS 3}

A REMAT utiliza o software de código livre Open Journal Systems (OJS) para gerenciamento e publicação da revista eletrônica. Para trazer melhorias no processo editorial e aperfeiçoar a experiência de uso e acesso dos usuários, os periódicos hospedados no Portal de Periódicos do IFRS realizaram o upgrade do sistema com a migração da versão OJS 2 para a versão OJS 3. Entre as principais novidades que terão maior impacto na REMAT, destacam-se: inscrição obrigatória do ORCID dos autores no cadastro de usuário do portal; adoção do framework Bootstrap; flexibilização do processo de editoração; possibilitação de discussões entre perfis e a viabilização dos 
usuários exercerem diversas funções dentro da revista sem a necessidade de criar outros cadastros ou alternar entre papéis no portal (BRITO et al., 2018). A descrição de cada uma dessas melhorias apresenta-se na Tabela 1. Essas informações foram compiladas de Brito et al. (2018).

Quadro 1. Atualizações no OJS 3.

\begin{tabular}{|c|c|}
\hline $\begin{array}{c}\text { Framework } \\
\text { Bootstrap }\end{array}$ & $\begin{array}{c}\text { Permite que páginas HTML adaptem-se a dispositivos móveis. Assim, em qualquer } \\
\text { dispositivo tem-se um visual com todas as funcionalidades por meio de temas } \\
\text { responsivos que podem ser personalizados. A técnica possibilita que o OJS } 3 \text { seja } \\
\text { responsivo aos atuais dispositivos. }\end{array}$ \\
\hline Editoração & $\begin{array}{c}\text { A atualização do sistema permite a flexibilização do processo de editoração. É possível } \\
\text { transitar entre as etapas no processo e personalizar o fluxo editorial. }\end{array}$ \\
\hline Discussões & $\begin{array}{l}\text { Visando aperfeiçoar o controle das comunicaçães, o OJS } 3 \text { tem a possibilidade de criar } \\
\text { uma discussão interna para cada uma das fases do fluxo editorial: submissão, revisão, } \\
\text { edição de texto e produção. Essa ferramenta permite que um avaliador crie um tópico } \\
\text { de discussão convidando um editor de seção, por exemplo. }\end{array}$ \\
\hline $\begin{array}{l}\text { Cadastro de } \\
\text { Usuários }\end{array}$ & $\begin{array}{l}\text { A integração entre o OJS } 3 \text { e o Open Researcher and Contributor ID (ORCID). O } \\
\text { cadastro de usuários em OJS } 3 \text { é interoperável com o ORCID, que é o identificador } \\
\text { digital do autor. Desta forma, ao se cadastrar no OJS } 3 \text { basta o usuário se conectar que } \\
\text { os demais campos do formulário de cadastro serão automaticamente preenchidos. }\end{array}$ \\
\hline Funções & $\begin{array}{c}\text { Usuários podem executar atividades pertencentes a dois papéis. O OJS } 3 \text { permite ao } \\
\text { Gerente da Revista criar novos papéis, ou mesmo renomear os existentes, sendo mais } \\
\text { fácil gerenciar os usuários do sistema dando as permissões necessárias a cada papel de } \\
\text { usuário nas instalações. Outra melhoria associada é a visualização de um painel único. } \\
\text { O usuário não precisa mudar de papel para realizar diferentes tarefas. }\end{array}$ \\
\hline
\end{tabular}

Fonte: Adaptação de Brito et al. (2018).

Com essas melhorias espera-se um melhor rendimento do fluxo editorial, facilitando o processo de submissão e editoração de artigos, bem como no processo de editoração, seja para autores, editores e avaliadores. Além disso, projeta-se a possibilidade de se operar o sistema totalmente pelo celular, o que pode tornar o conteúdo publicado mais acessível para os leitores, já que mais de $16 \%$ dos leitores da REMAT acessam a revista nesse tipo de dispositivo (VISSIRINI et al., 2019). 


\section{CONSIDERAÇÕES FINAIS}

Desde sua criação, a REMAT vem colhendo bons resultados em função de estar atenta às discussões realizadas pelos Editores da comunidade científica, e inovando em seus processos editoriais. O ano de 2019 é marcado um por crescente número de acessos e citações aos artigos da REMAT. Acredita-se que um dos fatores contribuidores tenha sido a inclusão da REMAT em indexadores e buscadores conhecidos pela comunidade acadêmica. Neste momento, a migração do OJS 2 para o OJS 3, realizada no mês de julho de 2019, traz um período de adaptação à nova interface do sistema, além de um novo leque de possibilidades, conforme descrito na seção anterior, que visam melhorar a interação entre autores, avaliadores e editores. Outra conquista deste ano que merece destaque foi a atribuição do DOI aos artigos da REMAT no mês de agosto, permitindo a identificação, localização e descrição unívoca dos artigos, e garantindo a propriedade intelectual aos autores.

\section{AGRADECIMENTOS}

Os autores agradecem o apoio financeiro concedido pela Pró-Reitoria de Pesquisa, Pós-graduação e Inovação (PROPPI) do IFRS por meio do Edital 01/2019/PROPPI - Apoio à Edição de Periódicos Científicos do IFRS.

\section{REFERÊNCIAS}

BRITO, R. F. et al. Guia do usuário do OJS 3. Brasília: Ibict, 2018. DOI: http://dx.doi.org/10.21452/978-85-7013-144-7.

CAMPANARIO, J. M. El sistema de revisión por expertos (peer review): muchos problemas y pocas soluciones. Revista española de documentación científica, v. 25, n. 3, p. 267-285, 2002.

COSTA, S. M. de S. Controle de qualidade em periódicos científicos eletrônicos disponibilizados na Internet: a questão do julgamento pelos pares. Revista de Biblioteconomia de Brasília, v. 20, n. 2, p. 227-236, jul./dez. 1996.

HARNAD, S. Implementing peer review on the net: scientific quality control in scholarly electronic journals. In: PEEK, R.; NEWBY, G. (Eds.) Scholarly Publication: The Electronic Frontier. Cambridge MA: MIT Press, 1996. Disponível em: http://cogprints.org/1692/1/harnad96.peer.review.html. Acesso em: 26. jun. 2019.

LANCASTER, F. W. The evolution of electronic publishing. Library Trends, v. 43, n. 4, p. 518-27, 1995.

PATRUS, R.; DANTAS, D. C.; SHIGAKI, H. B. Pesquisar é preciso. Publicar não é preciso: história e controvérsias sobre a avaliação por pares. Avaliação: Revista da Avaliação da Educação Superior, Campinas, v. 21, n. 3, p. 799-820, ago./nov. 2016. DOI: http://dx.doi.org/10.1590/S1414-40772016000300008 .

SILVA, L. H. R. da et al. Ações desenvolvidas na REMAT: Revista Eletrônica da Matemática com vistas à acessibilidade digital. Ciência da Informação em Revista, Maceió, v. 5, n. especial, p. 77-85, fev. 2018. DOI: http://dx.doi.org/10.21452/23580763.2018.5ne.77-85. 
VISSIRINI, C. C. et al. REMAT: Análise do perfil de seus usuários por meio da ferramenta google analytics e difusão do conhecimento científico entre professores e estudantes de matemática. Ciência da Informação em Revista, Maceió, v. 6, n. especial, p. 48-61, fev. 2019. DOI: http://dx.doi.org/10.21452/23580763.2019.6ne.48-61. 